\title{
A Global Robotic Telescope Network for Time-Domain Science
}

\author{
R. A. Street $^{1}$ and T. A. Lister ${ }^{1}$, Y. Tsapras ${ }^{1,2}$, A. Shporer ${ }^{1}$, \\ F. B. Bianco ${ }^{1}$, B. J. Fulton ${ }^{1}$, D. A. Howell ${ }^{1}$, B. Dilday ${ }^{1}$, M. Graham ${ }^{1}$, \\ D. Sand ${ }^{1}$, J. Parent ${ }^{1}$, T. Brown ${ }^{1}$, K. Horne ${ }^{3}$, M. Dominik ${ }^{3} \dagger$, \\ P. Browne ${ }^{3}$, C. Snodgrass ${ }^{4}$, N. Kains ${ }^{5}$, D. Bramich ${ }^{5}$, N. Law $^{6}$ and \\ I. Steele ${ }^{7}$ \\ ${ }^{1}$ LCOGT, 6740 Cortona Drive, Suite 102, Goleta, CA 93117, USA, \\ email: rstreet@lcogt.net \\ ${ }^{2}$ School of Mathematical Sciences, Queen Mary College, London E1 4NS, UK \\ ${ }^{3}$ SUPA, School of Physics \& Astronomy, University of St. Andrews, Fife, KY16 9SS, UK, \\ ${ }^{4}$ Max Planck Institute for Solar System Research, 37191 Katlenburg-Lindau, Germany \\ ${ }^{5}$ ESO, 85748 Garching bei München, Germany \\ ${ }^{6}$ Dept. of Astronomy and Astrophysics, University of Toronto, Ontario, M5S 3H4, Canada \\ ${ }^{7}$ Astrophysics Research Institute, Liverpool JMU, Birkenhead, CH41 1LD, UK.
}

\begin{abstract}
Las Cumbres Observatory Global Telescope Network (LCOGT) is currently building a new kind of general-purpose astronomical facility: a fully robotic network of telescopes of $2 \mathrm{~m}, 1 \mathrm{~m}$ and $0.4 \mathrm{~m}$ apertures and homogeneous instrumentation. A pan-network approach to scheduling (rather than per individual telescope) offers redundancy in the event of poor weather or technical failure, as well as the ability to observe a target around the clock. Here we describe the network design and instrumentation under development, together with the main science programmes already being lead by LCOGT staff.
\end{abstract}

Keywords. telescopes, instrumentation: photometers, instrumentation: spectrographs,stars: planetary systems, stars: supernovae

\section{Introduction}

At any given location, ground-based observations of time-domain phenomena suffer from gaps in their coverage caused by the diurnal cycle. The gaps can be supplemented with data from longitudinally-spaced sites, but at the cost of combining data from different instruments taken under very different conditions. While a number of such telescope networks like GONG and BiSON have been built to date, most have been designed for, and operated by, specific projects, with limited instrumentation. The Las Cumbres Observatory Global Telescope Network (LCOGT) will be a new, general-purpose facility for optical/NIR photometry and spectroscopy, which we describe in Sections $2 \& 3$. Designed from the outset for time-domain astronomy, the main science drivers for LCOGT staff are exoplanet and supernova science; we highlight these programmes in Sections $4 \& 5$.

\section{Robotic Observing Network}

Telescopes in the LCOGT network will be distributed longitudinally, at $\sim 6$ sites in both hemispheres, making it possible to observe around the clock. All telescopes will

$\dagger$ Royal Society University Research Fellow 
operate robotically as a cohesive whole, taking advantage of different sites to compensate for weather losses elsewhere. The network will incorporate 3 classes of telescope aperture: $2 \mathrm{~m}, 1 \mathrm{~m}$ and $0.4 \mathrm{~m}$. Each site will consist of a cluster of 2 or 31 -m telescopes accompanied by about 3 0.4-m telescopes. There will be at least 6 sites. The "Southern Ring" includes Siding Spring (Australia), the South African Astronomical Observatory and the Cerro Tololo Inter-American Observatory (Chile), while the "Northern Ring" will link sites at Maui (Hawai'i), Sedgwick (California), McDonald (Texas), Tenerife (Canary Islands) and a site in Asia yet to be selected. In addition to the 1-m and 0.4-m instruments we operate the existing two 2-m Faulkes Telescopes North (Hawai'i) and South (Australia). All telescopes of a given aperture class in the network will have an homogeneous instrument set and a consistent set of filters and calibration facilities. At present we have imaging cameras available at the Faulkes Telescopes; our plans for new instrumentation are described in Section 3. In partnership with SUPA/St. Andrews, LCOGT will be deploying its Southern Ring facilities first; construction is underway at CTIO and SAAO.

\section{New Instrumentation}

Our standard imaging instrument for the 1-m telescopes will be a SiNiSTRO camera, which consists of a Fairchild Imaging CCD $486 \mathrm{Bl}$ plus an LCOGT-designed controller. It will have a field of view (FOV) of $\sim 26^{\prime}$ with a pixel scale of $\sim 0^{\prime \prime} .4 /$ pixel.

To explore variable phenomena at very short time-scales by diffraction-limited imaging we are currently commissioning Lucky Imager, High Speed Photometer (LIHSP) cameras for the 2-m telescopes. They house an Andor iXon + DU888, $1 \mathrm{k} \times 1 \mathrm{k}$ back-illuminated frame-transfer EMCCD, and offer a FOV of up to $2^{\prime} .2 \times 2^{\prime} .2$ and observing cadences as fast as several tens of $\mathrm{Hz}$. The instruments will be exploited primarily for microlensingevent follow-up (Dominik 2010) and occultations, and are currently being tested. We will shortly begin testing an Andor Neo $2560 \times 2160$ sCMOS detector on a 1-m telescope, off-axis port; it has $0^{\prime \prime} .19$ pixels, a FOV of $360^{\prime \prime} \times 416^{\prime \prime}\left(\sim 6^{\prime} \times 7^{\prime}\right)$, and is capable of 100 fps at full frame.

The need for globally-networked spectographic facilities is even more acute than for imaging. Accordingly, LCOGT is developing spectrographs for our 2-m and 1-m telescopes. FLOYDS (Folded Low Order whYte-pupil Double-dispersed Spectrograph) will produce low-resolution ( $\mathrm{R} \sim 300-500)$ spectra between $\lambda 350-1100 \mathrm{~nm}$ once mounted on the 2-m telescopes. The science drivers include supernova classification, GRB follow-ups and AGN reverberation mapping.

Our 1-m telescopes will be equipped with MRES, our medium-resolution ( $\mathrm{R} \sim 25000)$ spectrograph which will cover a wavelength range of $\sim 390-860 \mathrm{~nm}$ and aims for a radialvelocity precision of $0.5 \mathrm{~km} \mathrm{~s}^{-1}$ for a $V=15 \mathrm{G} 0$ star with $v \sin i<3 \mathrm{~km} \mathrm{~s}^{-1}$.

\section{Exoplanet Science}

LCOGT runs a number of programmes to confirm exoplanet discoveries and characterise the systems. As members of the Palomar Transient Factory (PTF), and with team members collaborating with the WASP, Qatar Exoplanet Survey, HATNet and the CoRoT, Kepler teams, we have provided targeted high-precision photometry of discoveries from those surveys, contributing to the growing catalogue of exoplanets (Street et al. 2010; Lister et al. 2009). In targets of special interest we monitor transit timings long-term to search for variations (Fulton et al. 2011), and characterise long period/rare transit events such as those of HD 80606 b (Hidas et al. 2010; Shporer et al. 2010a). 
We operate the only fully-robotic follow-up programme for microlensing events, RoboNetII (Tsapras et al. 2009), combining our own facilities with those of the Liverpool Telescope, Canary Islands. This is a highly challenging programme: the $\sim 1500$ events discovered throughout a 6 -month season have to be prioritised dynamically as new data come in, and trigger observations in response to light-curve anomalies which betray the presence of planets. However, the planets are worth the effort, as this is the fastest way to find cool exoplanets in the outer reaches of their systems (Muraki 2011; Sumi et al. 2010).

\section{Supernova Science}

The supernova program at LCOGT obtains light curves of SNe discovered at the PTF and Pan-STARRS-1. Follow-up spectroscopy is obtained elsewhere, including Lick, Keck, Gemini and VLT, and (soon) with the low-resolution FLOYDS spectrographs on the Faulkes Telescopes. The SN programme includes:

- building a new sample of low-redshift SNe Ia to anchor the Hubble diagram measuring Dark Energy (Sullivan et al. 2011)

- investigating the progenitors of SNe Ia (Bianco et al. 2011)

- developing a better understanding of SNe Ia as cosmological probes (Conley et al. 2011; Cooke et al. 2011)

- following new classes of transients such as those too faint (Kasliwal et al. 2010) or too bright (Quimby et al. 2011) to fit into normal SN paradigms.

\section{Additional Science}

In addition to the main science programmes, LCOGT scientists collaborate widely and support a variety of other scientific programmes ranging from tracking Near-Earth Asteroids discovered by Pan-STARRS (resulting in 66 MPECS in 2011 to date) to the detection of the first detached double white-dwarf eclipsing binary (Steinfadt et al. 2010) and a ground-based detection of the beaming effect in that system (Shporer et al. 2010b).

\section{References}

Bianco, F. B., et al. 2011, ApJ, 741, 20

Conley, A., et al. 2011, ApJS, 192, 1

Cooke, J., et al. 2011, ApJ (Letters), 727, L35

Corsi, A., et al. 2011, arXiv:1101.4208

Dominik, M., 2010, General Relativity \& Gravitation, 42, 2075.

Fulton, B.J., et al. 2011, AJ, 142, 84.

Hidas, M. G., Tsapras, Y., Mislis, D., et al., 2010, MNRAS, 406, 1146.

Kasliwal, M. M., et al. 2010, ApJ (Letters), 723, L98

Lister, T. A., et al. 2009, ApJ, 703, 752.

Muraki, Y., Han, C., \& Bennett, D. P. 2011, ApJ, 741, 22.

Quimby, R. M., et al. 2011, Nature, 474, 487

Shporer, A., et al. 2010, ApJ, 722, 880.

Shporer, A., et al. 2010,ApJ (Letters), 725, L200.

Street, R. A., et al. 2010, ApJ, 720, 337.

Steinfadt, J.D.R., et al. 2010, ApJ (Letters), 716, L146.

Sullivan, M., et al. 2011, ApJ, 737, 102

Sumi, T., et al. 2010, ApJ, 710, 1641.

Tsapras, Y., et al. 2009, AN, 330, 4. 